\title{
Fixed implant-supported prosthetic rehabilitation in single tooth edentations - theoretical and practical aspects
}

\author{
Sorin Nicolae Popescu', Gabriela Tanase ${ }^{1}$, Gabriel Ciochindaa ${ }^{1}$, Augustin Mihai', \\ Viorel Stefan Perieanu', Ruxandra Stanescu', Iuliana Babiuc ${ }^{1}$, Radu Costea', \\ Simion Gh. Dumitru², Oana-Cella Andrei'1, Luminita Oancea², Mirel Toma', Mihai Burlibasa', \\ Corina Marilena Cristache ${ }^{2}$, Irina Adriana Beuran', Ileana Ionescu ${ }^{1}$ \\ 1"Carol Davila" University of Medicine and Pharmacy, Bucharest, Romania \\ ${ }^{2}$ Private dental practice, Bucharest, Romania
}

\begin{abstract}
Introduction. Reliable and rigorous, the new techniques and procedures of oral implantology have rapidly evolved, having an important place in the therapeutic plan elaboration, for the prosthetic restoration of different forms of edentation, especially single tooth edentation.

Purpose. Through this study we want to implement among the dental professionals, some notions of theoretical and practical character, regarding certain therapeutic implant-prosthetic flows for restoring single tooth edentations regardless of location.

Material and method. The study was carried out with the help of an 8-point questionnaire, applied to a study group composed of 45 dentists in Romania.

Resultsand discussion. The results of this study create a clear picture of how physicians know and apply the concepts related to implant supported prosthetic rehabilitation.

Conclusion. Implant-prosthetic rehabilitation of single tooth edentations is a safe treatment method, with few surgical complications and minimal bone loss, an aspect that also results from the answers of the doctors participating in the study.
\end{abstract}

Keywords: single tooth edentation, fixed implant-supported restorations, implant-prosthetic rehabilitation

\section{INTRODUCTION}

It is well known that, since the beginning of the 21 st century, there have been major changes in almost all areas, including in the medical field and dentistry must also be included in the medical field. In practice, in this field of dental medicine, the treatment methods and options offered to patients have evolved and are very rapidly evolving, taking into account that the patterns of dental disorders have changed. Recently, prevention programs have been introduced in Romania both in the elementary, middle and high schools (through the school cabinets), starting from elementary classes, and also in the private dental offices. The aim of these programs is to drastically reduce the frequency of the carious processes especially in children and, consequently, the decrease in the number of teeth lost, as a result of these carious processes not or incorrectly treated which, over time by their complications favors extraction (1-7).

Currently, patients have access to information and can benefit from both prevention programs and state-of-the-art treatments, which leads to a longer 
period of time keeping the teeth on the arch and a correct dispensing, after completing the prosthetic restoration in the dental office. Periodic checks that the patient must perform after the completion of a prosthetic restoration, regardless of its type (fixed or mobile), are mandatory, so that the restoration is optimal for as long as possible. If these controls are not performed on time, the long-term results may be compromised (1-7).

\section{PURPOSE}

But, it is known that, the most important factors in achieving an effective implant-prosthetic treatment, are the correct diagnosis and the optimal treatment option. As in any medical specialty, incorrect diagnosis can lead to choosing a wrong option of treatment and, by default, lead to failure. The correct diagnosis is made, by obtaining information directly from the patients and through a very rigorous clinical examination. These first two steps are performed in a first session, when the dentist must send the patient to make a set of x-rays, photographs and even a CT scan, when the insertion of dental implants is considered. In the same session the impression of the two dental arches is made, in order to analyze the situation on the resulting study models (1-7).

Thus, the treatment options possible in the case of edentations, and especially in the case of single tooth edentations, which is the theme of this study, can be chosen according to the clinical situation but, taking into account the requirements of the patients who naturally have a word to say. Most patients want fixed prosthetic restoration, as physionomic as possible, and less invasive, which do not require a long period of time to be manufactured and which, last but not least, are financially accessible, goal which is not easy to accomplish. However, it has been noticed that patients do not pay the same attention to lateral single tooth edentations and do not consider their prosthetic treatment to be necessary, unlike anterior edentations, when the physiognomic aspect is affected due to the lack of one or more teeth (1-7).

Almost always, there are several treatment options for single tooth edentations. The dentist must choose the correct treatment options, both according to the clinical situation of each individual case, and according to the requirements and possibilities of the patients. Although, almost all patients want a fixed prosthetic restoration, whether it is conventional or supported on dental implants, this variant cannot always be obtained. Before discussing the treatment plan with the patient, the dentist should evaluate the clinical and radiological situation to determine if a fixed implant-prosthetic rehabilitation is recommended for that type of single edentation (1-7). Specifically, starting from these principles mentioned above, we will try to implement among the dental practitioners, some notions of theoretical and practical character, regarding certain therapeutic implant-prosthetic flows of restoring of single tooth edentations regardless of location (maxillary, mandibular, in anterior or lateral areas), which is in fact the purpose of this study.

\section{MATERIALS AND METHODS}

Before taking the decision to perform any type of prosthetic restoration, including an implant-supported prosthetic restoration, according to the prosthetic practice guides developed in Romania, several factors must be taken into account, as follows, namely (1-7):

- The general health of the patients;

- Dental and periodontal status; dental occlusion and mandibular - cranial base relationship;

- The possibilities of maintaining oral hygiene;

- Patients' motivations and expectations;

- Complications that may limit clinical success;

- The advantages, disadvantages and longterm prognosis of prosthetic restorations;

- The cost of the entire treatment.

At the same time, before choosing an implant-prosthetic rehabilitation solution, in the case of a single tooth edentation, there are some criteria to be taken into account, namely (1-7):

- The age of the edentulous space;

- Localization of the edentulous space;

- The condition of the neighboring teeth;

- Bone supply.

Thus, implant supported single tooth restorations are indicated in patients with anodontia or those who have lost a tooth, following a trauma or other dental and periodontal disorders. The first analyses to be done are the general anamnestic data, as well as the investigation of the soft parts and the bone offer. Implant-supported restorations are performed in two stages: the first stage is the surgical one, and the second one is creating the fixed prosthetic restoration. At this time, dental abutments can be made of various materials, the most commonly used being titanium and titanium alloys, as well as zirconia (1-12). 
Even though in the medium and long term, dental implants offer a great biological advantage, however, there are conditions that contraindicate the dental implant. These could be classified into two major categories: local and general (1-7).

From the category of local contraindications for insertion of dental implants, it must be mentioned:

- Defective oral hygiene and the presence of inflammatory gingival outbreaks;

- Parafunctional activities;

- Patients with malignant tumors etc.

At the same time, as general complications of insertion of dental implants, it should be mentioned:

- Osteoporosis;

- Insulin-dependent diabetes;

- Excessive smoking;

- Alcohol abuse etc. (1-7)

There are several factors that influence the osseointegration of the dental implant, these being:

- Biocompatibility of the material;

- Dental implant design;

- The surface of the dental implant;

- The health state of the organism;

- Surgical implant insertion technique;

- Final prosthetic restoration (1-7).

Starting from these notions regarding the implant-prosthetic rehabilitation of the single tooth edentations, we developed a questionnaire based on 8 questions, which was then applied to a number of 45 dentists from several cities in Romania, including Bucharest, Brașov, Pitești, Giurgiu, Sibiu, Târgu Mureș, Alexandria etc., specialists familiar with the modern techniques of implant-prosthetic rehabilitation of single and/or multiple edentations. The study took place between June and September 2019. The dental practitioners included in the study were aged between 32 and 69 years, and their gender distribution was as follows: 29 of the subjects $(64.44 \%)$ were female while the rest of 16 dentists
(35.56\%) were male (Fig. 1). We opted for the questionnaire method, as it has a number of advantages, such as:

- The speed and convenience of obtaining guidance information for prospecting purposes;

- Trends of opinion;

- Sincerity of the answers, due to anonymity and confidentiality;

- Capture the influence of a facilitating and disruptive factor, before the effects are factually visible.

\section{Questionnaire}

1. The factors to be taken into account when performing a prosthetic restoration, including a fixed implant-supported prosthetic restoration are? a. The general health status of the patients, the dental and periodontal status, the dental occlusion and the mandibular - cranial base relationship; b. Possibilities to maintain a good oral hygiene, motivations and expectations of patients, complications that may limit clinical success; c. Advantages, disadvantages and long-term prognosis of prosthetic restorations, the cost of the entire treatment. Correct answers: $a, b, c$.

2. The criteria to be considered before choosing an implant-prosthetic rehabilitation solution, in the case of single tooth edentation, are? a. The age of the edentulous space; $\boldsymbol{b}$. Localization of the edentulous space; c. The condition of the neighboring teeth; $\boldsymbol{d}$. Bone supply; $\boldsymbol{e}$. The technical and material endowments of the dental office that perform the implant-prosthetic rehabilitation. Correct answers: $\boldsymbol{a}, \boldsymbol{b}, \boldsymbol{c}, \boldsymbol{d}$.

3. Single tooth implant-supported prosthetic restorations are indicated in? $\boldsymbol{a}$. Patients with anodontia; b. Patients who have lost a tooth due to trauma or other dental and/or periodontal disorders; c. Patients with severe heart disease who do

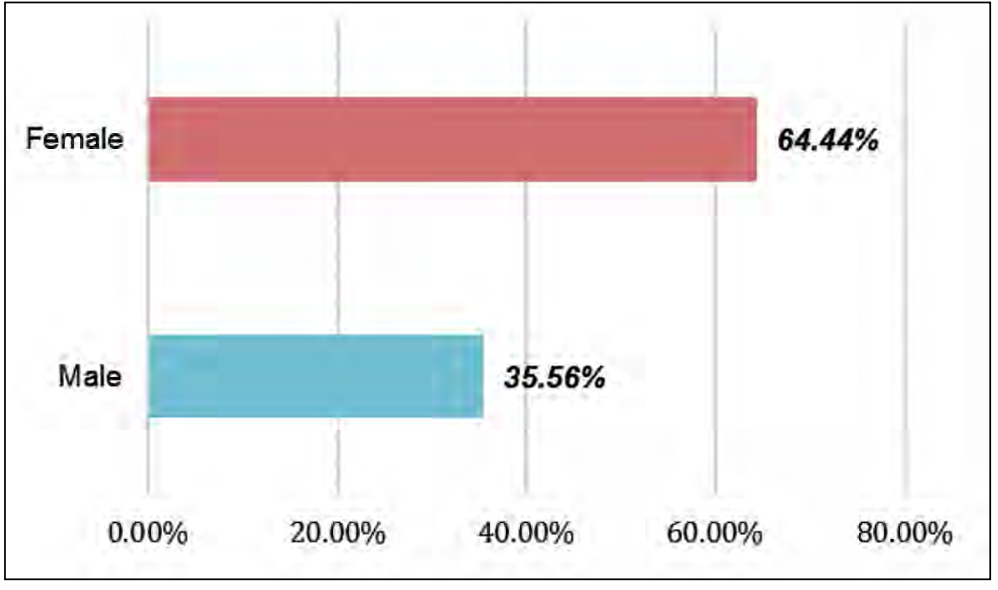

FIGURE 1. Gender distribution of study participants 
not support other therapeutic solutions. Correct answers: $\boldsymbol{a}, \boldsymbol{b}$.

4. The stages of performing the implant-supported restorations are? $\boldsymbol{a}$. The psychological preparation stage of the patient with this idea; $\boldsymbol{b}$. The stage of creating in advance of the future implant-supported restoration; c. Surgical stage; d. Creating the fixed prosthetic restoration. Correct answers: $c, d$.

5. In the case of dental implants, the materials from which prosthetic abutment are made can be? a. Titanium and titanium alloys; $\boldsymbol{b} . \mathrm{Ni}-\mathrm{Cr}$ and $\mathrm{Cr}$ Co alloys; c. Zirconia; d. Copper-based alloys from the bronze category. Correct answers: $\boldsymbol{a}, \boldsymbol{c}$.

6. In the category of local contraindications for inserting dental implants, the following are included? a. Defective oral hygiene and the presence of inflammatory gingival outbreaks; $\boldsymbol{b}$. Parafunctional activities; $\boldsymbol{c}$. Patients with malignant tumors; $d$. Patients with failed implant-supported restorations in the background. Correct answers: $\boldsymbol{a}, \boldsymbol{b}, \boldsymbol{c}$.

7. In the category of general contraindications for inserting dental implants, the following are included? a. Osteoporosis; $\boldsymbol{b}$. Insulin-dependent diabetes; c. Excessive smoking; d. Alcohol abuse; $\boldsymbol{e}$. Stress. Correct answers: $\boldsymbol{a}, \boldsymbol{b}, \boldsymbol{c}, \boldsymbol{d}$.

8. There are several factors that influence the osseointegration of the dental implant, these being? $a$. The biocompatibility of the material, the design and the surface of the implant; $\boldsymbol{b}$. The health state of the organism; c. Surgical implant insertion technique; $\boldsymbol{d}$. Final prosthetic loading. Correct answers $a, b, c, d$.

\section{RESULTS AND DISCUSSION}

Regarding the factors to be taken into account when performing a prosthetic restoration, most of the study participants 39 (representing $86.67 \%$ ) an- swered correctly - variants a, b and c. Only 6 participants (representing 13.33\%) excluded from the correct variants the answer related to the advantages, disadvantages and long-term prognosis (Fig. 2).

All the specialists included in the study answered correctly (variants $a, b, c$ and d) to the question related to the selection criteria for choosing an implant-prosthetic rehabilitation solution.

The answers to the third question show us that the vast majority of the respondents (42 representing $93.33 \%$ ) know very well the indications of implant-supported single tooth prosthetic restorations (variants a and b). Only 3 respondents mistakenly included among the answers the variant related to patients with severe heart disease (Fig. 3).

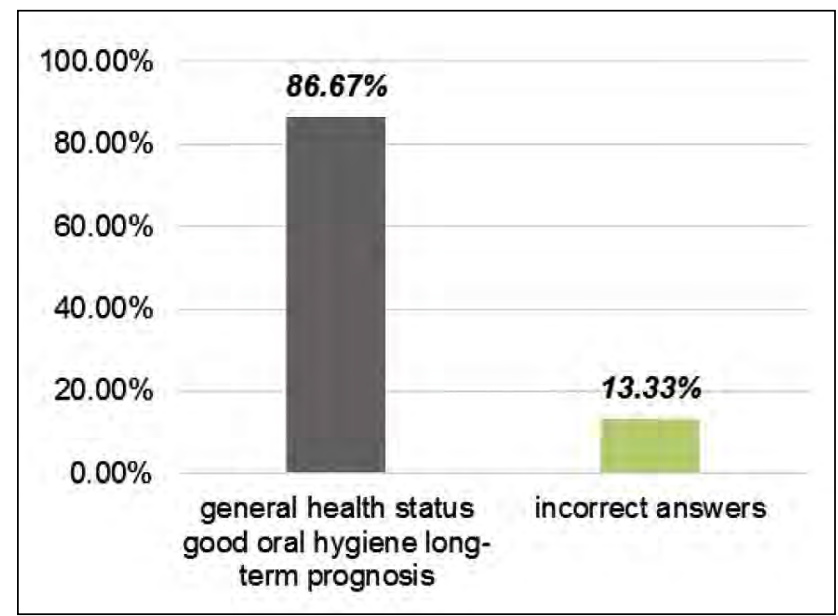

FIGURE 2. Knowledge of the factors that matter in performing prosthetic restorations

In relation to the stages of the implant-supported restorations, all the specialists included in the study have answered correctly, namely the surgical and the prosthetic stage.

About the prosthetic connection to dental implants, most of the subjects (32 representing $71.11 \%$ ) answered correctly (titanium or zirconium

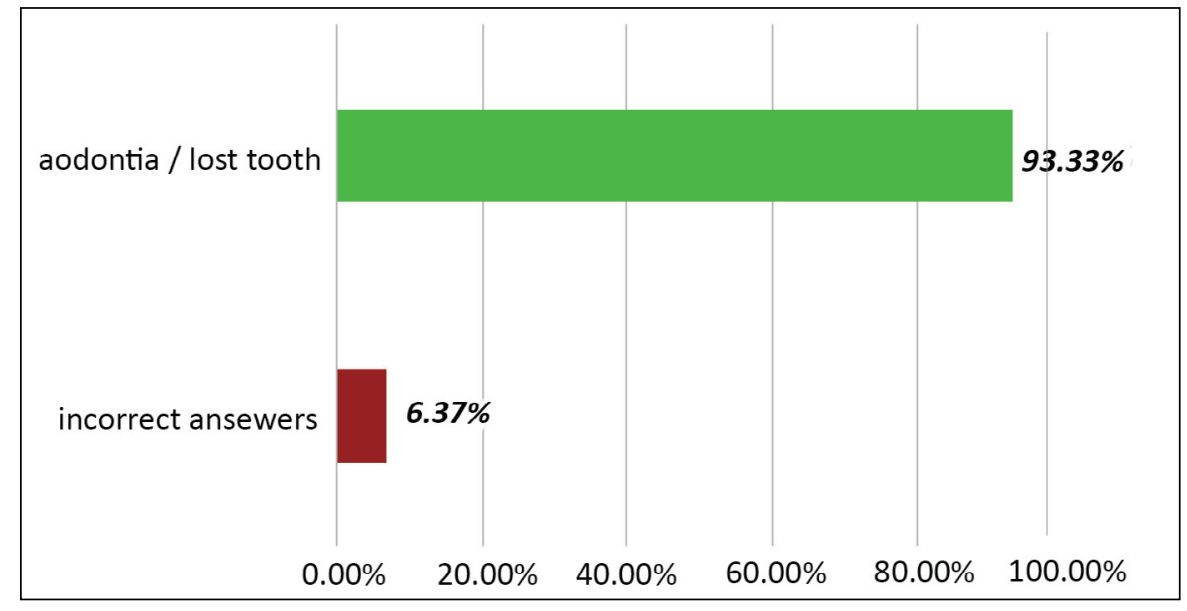

FIGURE 3. Indications of implantsupported single tooth prosthetic restorations 


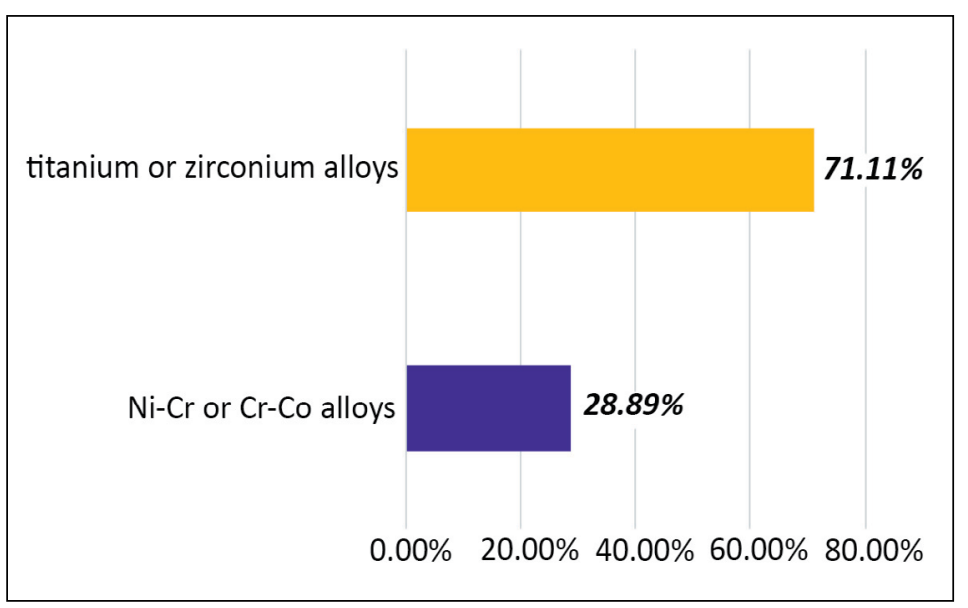

FIGURE 4. Recommended prosthetic connections for dental implants alloy abutments) and only 13 subjects (representing $28.89 \%$ ) answered incorrectly, adding also the abutments based on Ni-Cr and Cr-Co (Fig. 4).

Concerning the local contraindications for inserting dental implants, the vast majority of the doctors included in the study (43 representing 95.56\%) answered correctly - variants a, b and c (hygiene, parafunctional activities and tumors). Only 2 physicians included in the study responded incorrectly, including failures of previous implant-prosthetic restorations (Fig. 5).
Regarding the general contraindications for dental implants 38 of the subjects (representing 84.44\%) answered correctly 2 variants a, b, c and d. The remaining 7 subjects (representing 15.56\%) included besides the correct response variants the stress (Fig. 6).

To the last question in the study regarding the factors that influence the osseointegration of the dental implant, all the specialists included in the study answered correctly - variants a, b, c and d (biocompatibility, health state of the body, surgical technique and final prosthetic loading).
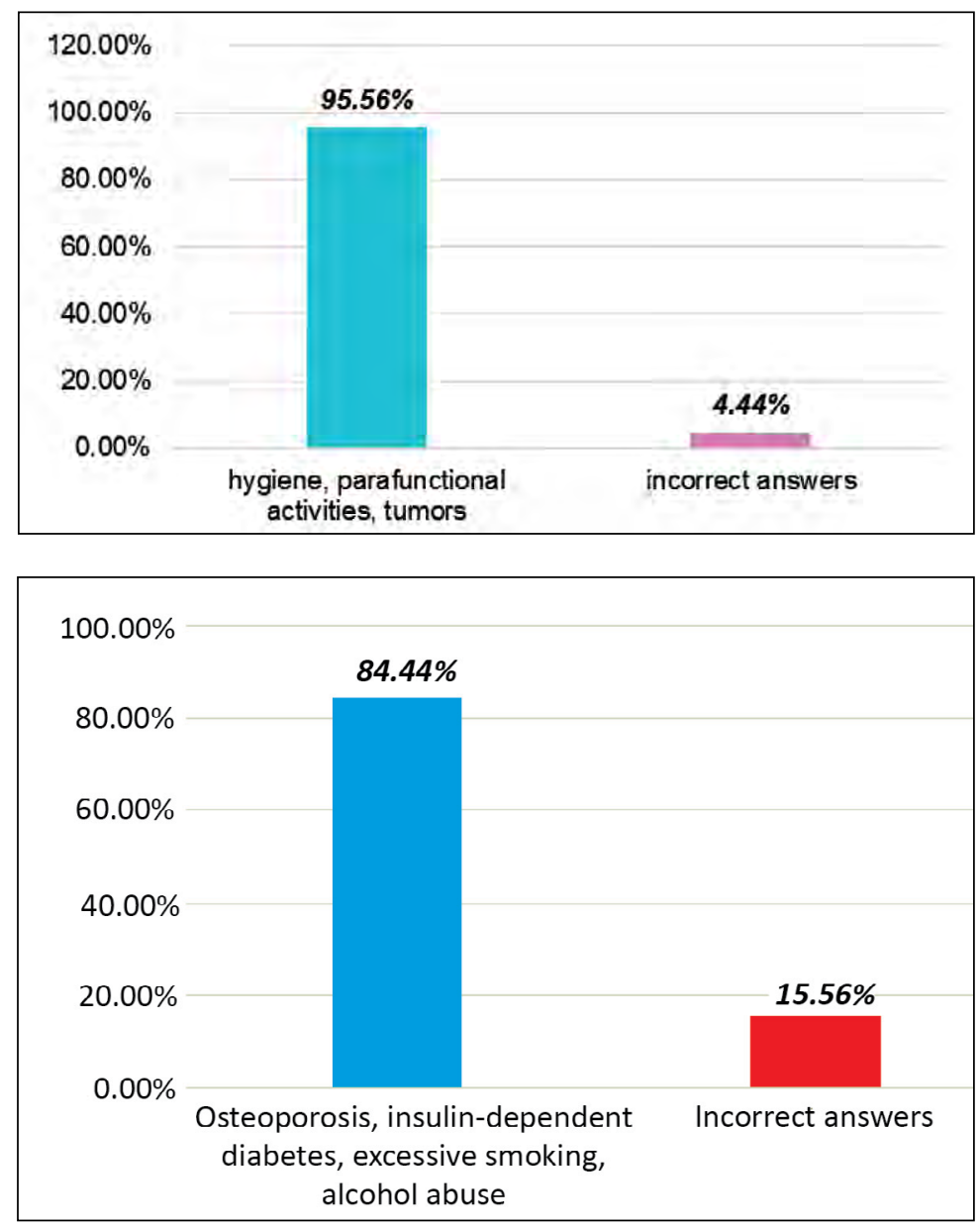

FIGURE 5. Knowing the local contraindications of dental implants
FIGURE 6. General contraindications for dental implants 


\section{CONCLUSIONS}

As a result of the answers to the 8 questions, we can conclude the following aspects, as follows:

Most of the dental practitioners participating in the study (over $80 \%$ ) possess firm theoretical and practical knowledge regarding the implant-prosthetic rehabilitation of single tooth edentations. The exception is that situation in which almost 30\% of the subjects made a regrettable confusion regarding the materials from which the implant prosthetic abutments are made, indicating besides titanium, titanium alloys, zirconium, and $\mathrm{Ni}-\mathrm{Cr}$ and Cr-Co based alloys.

Osseointegration and maintenance of dental implants are also influenced by other factors, such as age, diet, medications, system disorders and oral disorders.

Implant-prosthetic oral rehabilitation can be a very good treatment solution for healthy patients who want to replace missing teeth and can undergo surgery.

When planning the insertion of a dental implant, at least 5 factors must be taken into account, which can be more difficult to influence later, in the prosthetic stage: the position of the dental implant; diameter of dental implant; the axis tilt angle of the dental implant; contour of peri-implant soft tissues; intragingival position of the shoulder of the dental implant.
In general, high success rates have been reported for the restoration of a single tooth, especially for the replacement of the anterior teeth. Regarding the replacement of the posterior teeth, this is more problematic, especially in the case of the molars, due to the size discrepancy between the implant and the tooth, and the high occlusal stress.

Both from our experience, of the authors, as well as studying the specialized literature, we can say with certainty that, the implant-prosthetic rehabilitation of single tooth edentations represents a safe treatment method, with few surgical complications and with minimal bone loss.

Implant-prosthetic rehabilitation of single tooth edentations is a valuable treatment option, with a fair cost-benefit ratio, especially in situations where the teeth neighboring the edentulous space are integral, and the patient is young and healthy.

In the case of anterior edentations, after the insertion of a dental implant, provisional prosthetic restorations can be used, which restore the physiognomy. Partial acrylic prostheses may be used which do not press on the area of the dental implant or maryland bridge that attach to the neighboring teeth, without requiring their preparation.

\section{Acknowledgement}

In this article, all the authors have equal contribution with the first author.

Conflict of interest: none declared Financial support: none declared

\section{REFERENCES}

1. Dina MN, Andrei OC, lonescu I et al. Aspecte teoretice și practice în tratamentul clasic și modern al edentației unidentare. In: Tănase G, Dina MN, Dumitru SGh et al. Probleme în medicină și biologie Vol. IX. București: Ed. Ars Docendi, 2019:165-312.

2. Forna NC, Trăistaru T. Ghid de practică în protetica dentară, 2010; 353.

3. Gall II. Asistența stomatologică. București: Ed. Didactică și Pedagogică, 1971.

4. Miyasaki-Ching CM. Elemente clinice de stomatologie. București: Ed. All Educational, 2001.

5. Zarnea L. Pedodonție. București: Ed. Didactică și Pedagogică, 1993.

6. Rosenstiel SF, Land MF, Fujimoto J. Contemporary fixed prosthodontics. 4th Edition. St. Louis: Mosby Elsevier, 2006.

7. Anusavice KJ. Dental materials. Philips' Science 11th Edition. St. Louis: Saunders Elsevier, 2003.
8. Ispas DC, Eftene OA, Burlibașa M et al. Implications of titanium in orthodontics and dental facial orthopedics. Metalurgia International. 2011; 16(10):72-74.

9. Burlibașa M, Cernușcă-Mițariu M, Cernușcă-Mițariu S et al. Theoretical and practical aspects related to biomaterials decontamination in dental medicine (with reference to dental prosthetics). Metalurgia International. 2013; 18(4):261-267.

10. Burlibasa L, Domnariu C. Epigenetic landscape of human diseases. Acta Medica Transilvanica. 2018; 23(2):33-37.

11. Bodnar DC, Burlibașa L, Vârlan $C$ et al. Mercury, biocompatibility and its impact on environment. Metalurgia International. 2009; 14:95-100.

12. Cristache $\mathrm{CM}$, Burlibașa $\mathrm{M}$, Cristache $\mathrm{G}$ et al. Zirconia and its biomedical applications. Metalurgia International. 2011; 16(7):18-23. 